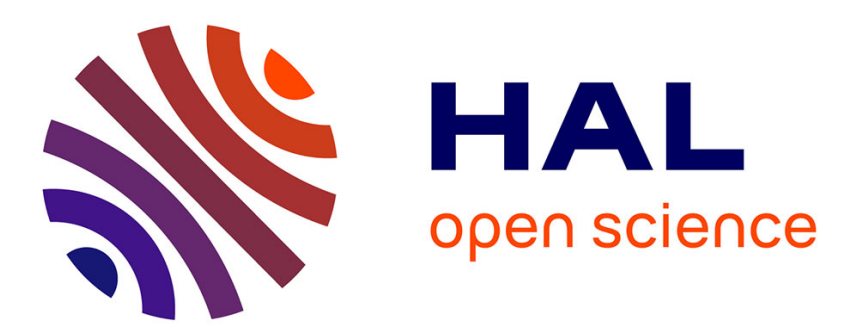

\title{
Three dimensional magnetic field mathematical model and its applications to calculation of fields and losses in turbogenerator end regions
}

Ya. B. Danilevich, V.I. Yakovlev

\section{- To cite this version:}

Ya. B. Danilevich, V.I. Yakovlev. Three dimensional magnetic field mathematical model and its applications to calculation of fields and losses in turbogenerator end regions. Revue de Physique Appliquée, 1990, 25 (7), pp.661-667. 10.1051/rphysap:01990002507066100 . jpa-00246230

HAL Id: jpa-00246230

https://hal.science/jpa-00246230

Submitted on 1 Jan 1990

HAL is a multi-disciplinary open access archive for the deposit and dissemination of scientific research documents, whether they are published or not. The documents may come from teaching and research institutions in France or abroad, or from public or private research centers.
L'archive ouverte pluridisciplinaire HAL, est destinée au dépôt et à la diffusion de documents scientifiques de niveau recherche, publiés ou non, émanant des établissements d'enseignement et de recherche français ou étrangers, des laboratoires publics ou privés. 


\title{
Three dimensional magnetic field mathematical model and its applications to calculation of fields and losses in turbogenerator end regions
}

\author{
Ya. B. Danilevich and V. I. Yakovlev \\ Vniielectromash, Dvortzovaya nat., 18, Leningrad, U.S.S.R. \\ (Reçu le 3 octobre 1989, révisé le 3 avril 1990, accepté le 23 avril 1990)
}

\begin{abstract}
Résumé. - On développe une méthode de calcul numérique tridimensionnelle du champ magnétique à l'intérieur et à la surface des têtes de bobines de gros générateurs. La méthode tient compte de la distribution des conducteurs à la périphérie. Les conducteurs du bobinage stator sont décrits par deux couches de charges magnétiques situées sur les surfaces inférieures et supérieures, ce qui réduit considérablement la région occupée par ces charges. On fournit les résultats du calcul dans le cas d'un turbogénérateur.
\end{abstract}

\begin{abstract}
Development of three dimensional mathematical model of electromagnetic field is of great importance for turbogenerators, especially for those with large ratings or little active volume [1, 2]. Such a mathematical model permits us to carry out accurate numerical investigation of the generator design instead of creating a physical model much less correct and much more costly.
\end{abstract}

\section{The mathematical model.}

Three dimensional electromagnetic field consideration is the most important when the end packet teeth region where the stator winding bars are outcoming from the slots is investigated. In this region the resultant magnetic field is the combination of magnetic fields which are originated from different sources and which differ in amplitude and phase.

Determination of the fields is performed in two stages. At the first stage two-dimensional fields with the periodical changing of the field along the angular

real configuration of the end zone, eddy currents in the press flange, generator operation parameters and saturation effect of the active zone are taken into account. The results of calculations are used as boundary conditions for determination of the components of three-dimensional fields. The region where the field is determined at the second stage is shown in figure 2. It is necessary to mention that the region under consideration at the second stage is not uniform both from the magnetic and electrical points.
1.1 TWO-DIMENSIONAL MODEL CONSIDERATION (the first stage, Fig. 1). - The problem is to be solved with the following assumptions:

- non uniform slotted stator and rotor structures are replaced by uniform ones with magnetic permeabilities $\mu_{r}, \mu_{\varphi}, \mu_{z}[2,5]$;

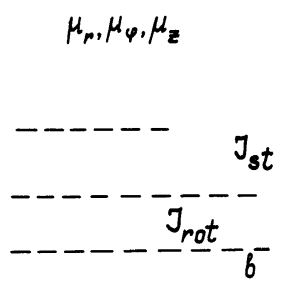

$d_{2}$ 0

Fig. 1. - The region for determining two-dimensional fields. 


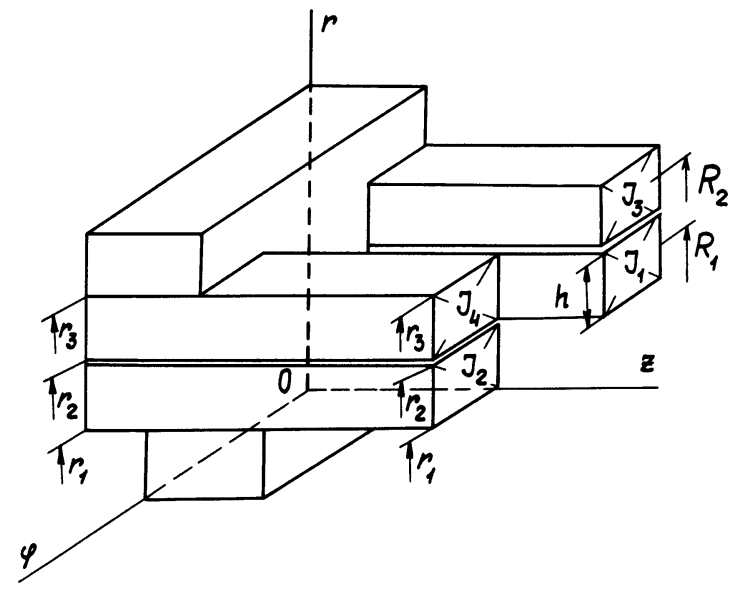

Fig. 2. - The region for determining three-dimensional fields.

- the currents in stator and rotor windings are represented by current layers with known densities, the currents phase difference being taken into account ;

- the values of magnetic permeabilities are taken from the results of the machine magnetic circuit calculation ;

- the magnetic field higher harmonics are not considered.

The electromagnetic field in the region will satisfy the following equation for the scalar magnetic potential (Appendix 1)

$$
\begin{aligned}
\frac{1}{r} \frac{\partial}{\partial r}\left(r \mu_{r} \frac{\partial U_{\mathrm{II}}}{\partial r}\right) & +\frac{\partial}{\partial r}\left(\mu_{z} \frac{\partial U_{\mathrm{II}}}{\partial z}\right)+ \\
& +\frac{1}{r^{2}} \frac{\partial}{\partial \varphi}\left(\mu_{\varphi} \frac{\partial U_{\mathrm{II}}}{\partial \varphi}\right)=-\rho_{\mathrm{II}},
\end{aligned}
$$

where $\mu_{r}, \mu_{\varphi}$ and $\mu_{z}$ are the components of stator core magnetic permeability tensor;

$\rho_{\text {II }}-$ volume density of the magnetic charges.

Inside the laminated stator core $\mu_{r}, \mu_{\varphi}$ and $\mu_{z}$ are of different values and not equal to $\mu_{0}$.

Magnetic charges can be found from stator current density $\mathcal{F}_{s t}$ using $H_{0 \mathrm{II}}$ - vector potential of the current, see Appendix 2. In the region of Fig. 2 only the axial component $\mathcal{F}_{z}$ exists, and

$$
\int_{0}^{2 \pi} y_{z} r \mathrm{~d} \varphi=0
$$

From (2) it follows that

$$
\bar{H}_{0 \mathrm{II}}=\bar{\ell}_{r} H_{0 r \mathrm{II}}=-\bar{\ell}_{r} \int_{0}^{\varphi} \gamma_{z} r \mathrm{~d} \varphi
$$

and the magnetic charge density is

$$
\rho_{\mathrm{II}}=-\operatorname{div} \mu \bar{H}_{0 \mathrm{II}}=-\frac{1}{r} \frac{\partial}{\partial r}\left(r \mu_{r} H_{0 r \mathrm{II}}\right) .
$$

The section $\varphi=0$ is chosen in such a way that $\gamma_{z}(\varphi)$ is an even function.

When conductive elements are considered, equation (1) must be solved together with the equation

$$
\operatorname{rot} \frac{1}{\gamma} \operatorname{rot} \dot{\bar{H}}_{0 \mathrm{II}}+j \omega \mu \dot{\bar{H}}_{0 \mathrm{II}}=j \omega \mu \operatorname{grad} \dot{U}_{\mathrm{II}}
$$

where $\gamma-$ is conductivity of the material. For the known values of $\dot{\bar{H}}_{0 \mathrm{II}}$ and $\dot{U}_{\mathrm{II}}$ it is possible to determine magnetic field strength. $\dot{\bar{H}}_{\text {II }}=\dot{\bar{H}}_{0 \mathrm{II}}-\operatorname{grad} \dot{U}_{\mathrm{II}}$, eddy currents $\dot{\bar{\delta}}=\operatorname{rot} \dot{\bar{H}}_{0 \mathrm{II}}$ and local losses

$$
Q=\frac{1}{2 \gamma} g F^{*} \text {. }
$$

It is possible to show [4] that the rectangular stator bar can be replaced by two layers of charges distributed on upper and lower bar surfaces with surface charge density

$$
\sigma=-\operatorname{Div} \mu \bar{H}_{0},
$$

or $\sigma_{\text {up }}=\mu_{r} H_{0 r} \quad$ on the upper and

$\sigma_{\text {low }}=-\mu_{r} H_{0 r}$ on the lower surfaces.

In order to simplify numerical calculations it is desirable to assume that stator bar is not rectangular but curvilinear quadrangle with current density distribution $\gamma_{z}=\gamma_{\mathrm{st}} \frac{R_{\mathrm{mit}}^{2}}{r^{2}}$ (Fig. 3). In this case

$$
\begin{aligned}
\sigma_{\mathrm{up}} & =-\mu_{r} \frac{\mathcal{J}_{\mathrm{st}} R_{\mathrm{mit}}^{2}}{r_{\mathrm{up}}} \varphi, \\
\sigma_{\text {low }} & =\mu \frac{\digamma_{\mathrm{st}} R_{\mathrm{mit}}^{2}}{r_{\text {low }}} .
\end{aligned}
$$

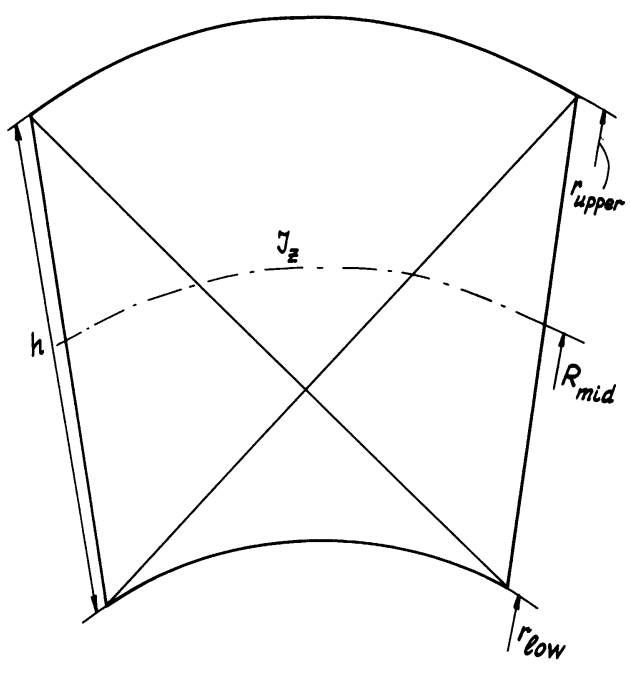

Fig. 3. - Cross section of the stator winding bar. 
The distribution of $g_{z}(\varphi)$ is a piecewise-linear function (Fig. 2), therefore at the end region sections where $g_{z} \neq 0$ we shall have $H_{0 r}(\varphi)=A+B \varphi$ and between stator bars $H_{0 r}(\varphi)=$ const. The function $H_{0 r}(r)$ is piecewise-constant and from (6) it follows that the relationship $\sigma(\varphi)$ will have the same character as $H_{0 r}(\varphi)$. As a result, a two-layer stator winding can be replaced by magnetic charges situated at three surfaces (Fig. 4).

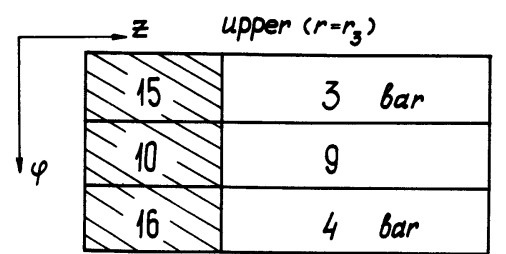

\begin{tabular}{|l|l|}
\hline 17 & midlle $\left(r-r_{2}\right)$ \\
\hline 12 & 5 bar \\
\hline 18 & 11 \\
\hline 18 & 6 \\
\hline
\end{tabular}

\begin{tabular}{|l|l|}
\hline 13 & Cower $\left(r=r_{p}\right)$ \\
\hline & 1 bar \\
\hline $\begin{array}{l}\text { Stator } \\
\text { core }\end{array}$ & $\begin{array}{c}\text { End parts of the } \\
\text { wihding }\end{array}$ \\
\hline
\end{tabular}

Fig. 4. - The surfaces where magnetic charges which substitute the two-layer winding are distributed.

Choosing the boundaries of the zone along the coordinate $\varphi$ in the most convenient way ( $\varphi_{\mathrm{B}} \leqslant \varphi \leqslant \varphi_{\text {end }}$ ) where $\varphi_{\mathrm{b}}$ is the coordinate of the zone beginning and $\varphi_{\text {end }}$ - that of zone finishing (Fig. 5), we obtain :

$$
\begin{aligned}
H_{0 r}(\varphi)=-\int_{\varphi \mathrm{b}}^{\varphi} \gamma_{z} r \mathrm{~d} \varphi= \\
\quad=-\gamma_{\mathrm{II}} r \sin \varphi_{\mathrm{b}}-\int_{\mathrm{B}}^{\varphi} \gamma_{z} r \mathrm{~d} \varphi .
\end{aligned}
$$

The first member of (8) corresponds to the harmonic distribution of current density along $\varphi$ and $\gamma_{\text {II }}$ is the amplitude of $\gamma_{z}$ when a twodimensional field is considered.

In Appendix 3 there are given the formulae for magnetic charges $\sigma_{i}(\varphi)=A_{i}+B_{i}(\varphi)$. The region under consideration (Fig. 4) is divided into 18 parts which differ by the values of coefficients $A_{i}$ and $B_{i}(i=1,2, \ldots, 18)$. The geometric quantities used are clear from figure $5 ; y_{1}, y_{2}, g_{3}$ and $f_{4}$ are current densities in stator bars.

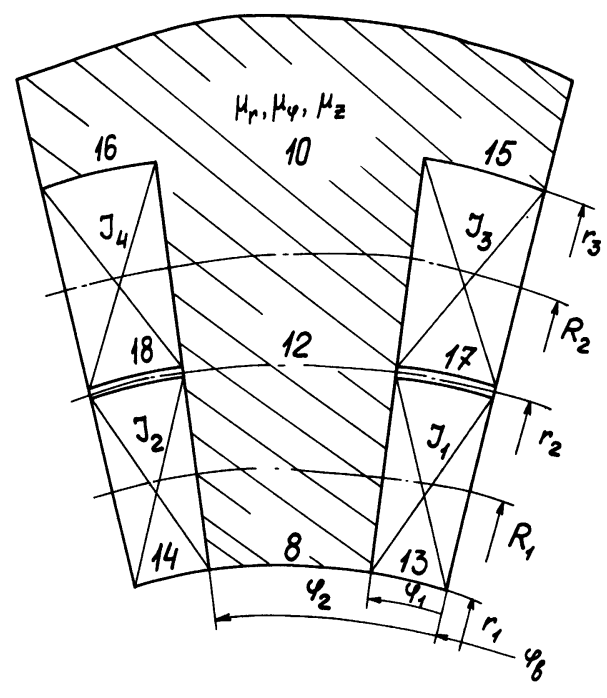

Fig. 5. - Cross section of the three-dimensional region.

1.2 THREE-DIMENSIONAL MODEL (the second stage, Fig. 2). - It has been mentioned before that for the three-dimensional model the boundary conditions for scalar magnetic potential (Eq. 1) are determined from solution of the two-dimensional problem assuming that the relationship of the components depending on $\varphi$ is harmonic. In this case the stator $\bar{g}_{\text {st }}$ and rotor $\bar{g}_{\text {rot }}$ current densities are :

$$
\begin{aligned}
\bar{g}_{\text {st }}(t, \varphi) & =\bar{g}_{\text {st }}(r, z) \cos \left(\omega t-\varphi-\varphi_{0}\right) \\
& =R_{\mathrm{e}}\left[\bar{\gamma}_{\text {st }}(r, z) \mathrm{e}^{j\left(\omega t-\varphi-\varphi_{0}\right)}\right] \\
\bar{g}_{\text {rot }}(t, \varphi) & =\bar{g}_{\text {rot }}(r, z) \cos (\omega t-\varphi) \\
& =R_{\mathrm{e}}\left[\bar{g}_{\text {rot }}(r, z) \mathrm{e}^{j(\omega t-\varphi)}\right],
\end{aligned}
$$

where $\varphi_{0}$ is the displacement angle between stator and rotor currents. Introducing a system of rotating coordinates, e.g. replacing $\varphi$ by $-\omega t+\varphi$, we shall have

$$
\begin{aligned}
\bar{F}_{\text {rot }}(\varphi) & =\bar{g}_{\omega t}(r, z) \cos (-\varphi) \\
& =R_{\mathrm{e}}\left[\bar{F}_{\text {rot }}(r, z) \mathrm{e}^{-j \varphi}\right] \\
\bar{\gamma}_{\text {st }}(\varphi) & =\bar{g}_{\text {st }}(r, z) \cos \left(-\varphi-\varphi_{0}\right) \\
& =\mathrm{e}^{\text {st }},-\cdot+
\end{aligned}
$$

Using (9) it is possible to determine

$$
\begin{aligned}
H_{0 r}(\varphi)=r \int_{-\varphi_{0}}^{\varphi} \bar{\gamma}_{z} & \cos \left(\varphi+\varphi_{0}\right) \mathrm{d} \varphi= \\
& =R_{\mathrm{e}}\left[r \mathcal{F}_{z} \mathrm{e}^{-j\left(\varphi+\varphi_{0}+\frac{\pi}{2}\right)}\right]
\end{aligned}
$$

at the zone of stator current. The same expression can be used at the zone of rotor current when $\varphi_{0}=0$. 
Taking into account (10) the solution of (1) can be found in the form

$$
\begin{aligned}
\mho_{\mathrm{II}}(r, \varphi, z) & =\mho(r, z) \cos \left(\varphi+\varphi_{0}+\frac{\pi}{2}\right)= \\
& =\mathrm{R}_{\mathrm{e}}\left[\mho(r, z) \mathrm{e}^{-j\left(\varphi+\varphi_{0}+\frac{\pi}{2}\right)}\right] .
\end{aligned}
$$

If expressions (10) and (11) are substituted into (1) the multiplier $\cos \left(\varphi+\varphi_{0}+\frac{\pi}{2}\right)$ is reduced. Therefore there is no need to solve equation (1) for every value of $\varphi$ and the solution in the form $\mathcal{V}(r, z)$ corresponds to $\vartheta_{\text {III }}(r, \varphi, z)$ when $\varphi=-\varphi_{0}-\frac{\pi}{2}$. At every other section the distribution of $\mathcal{V}$ is determined according to (11).

In this paper the linear problem is considered and the resultant field can be found as the sum of the fields caused by stator and rotor currents.

$$
\begin{aligned}
& \bigcup_{\text {III }}(r, \varphi, z)=\mathcal{V}_{\text {rot }}(r, z) \cos \left(\varphi+\frac{\pi}{2}\right)+ \\
& +\vartheta_{\mathrm{st}}(r, z) \cos \left(\varphi+\varphi_{0}+\frac{\pi}{2}\right) \\
& =R_{\mathrm{e}}\left\{\mathrm{e}^{-j\left(\varphi+\frac{\pi}{2}\right)}\left[\mathcal{\vartheta}_{\text {rot }}(r, z)+\vartheta_{\mathrm{st}}(r, z) \mathrm{e}^{-1 \varphi_{0}}\right]\right\} \\
& =R_{\mathrm{e}}\left[\mathrm{e}^{-j\left(\varphi+\frac{\pi}{2}\right)} \cdot \dot{\mathcal{V}}_{\mathrm{II}}\right] \text {. }
\end{aligned}
$$

In (12) scalar potential $\dot{v}_{\text {II }}$ is the solution for twodimensional field at the region of end packets of stator core.

The function $\dot{H}_{0 r}$ which according to (4) deter-

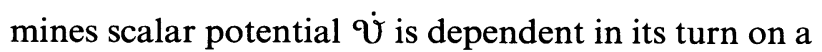
complex current density

$$
\dot{\gamma}_{z}=g_{\text {rot } z}+\gamma_{\text {st } z} \mathrm{e}^{-j \varphi_{0}} .
$$

The expressions (12) and (13) permit to obtain $\bigcup_{\text {III }}(r, \varphi, z)$ through $\bigcup_{\text {II }}(r, z)$ in every section $\varphi$, considering the region of stator currents and replacing for this reason $\varphi$ by $\varphi-\varphi_{0}$ in section $\varphi-\varphi_{\mathrm{b}}$

$$
\vartheta_{\mathrm{III}} / \varphi_{\mathrm{b}}=R_{\mathrm{e}}\left[\mathrm{e}^{j\left(\varphi_{0}-\frac{\pi}{2}-\varphi_{\mathrm{b}}\right)} \cdot \dot{\mho}_{\mathrm{II}}\right]
$$

Expression (14) is used as a boundary condition for determining three-dimensional solution for $\vartheta_{\text {III }}(r, \varphi, z)$ in the region shown in figure 2 .

\subsection{NUMERICAL REALIZATION OF THE METHOD. -} The problem is solved numerically using finite difference method. Application of this method is associated with some calculating difficulties. In order to overcome them it is proposed to use a uniform conservative difference scheme on seven-point pattern (Fig. 6), and to provide that in every node the following condition is fulfilled :

$$
\rho_{i} \Delta V_{i}=\int_{\Delta V_{i}} \rho(r, \varphi, z) \mathrm{d} V
$$

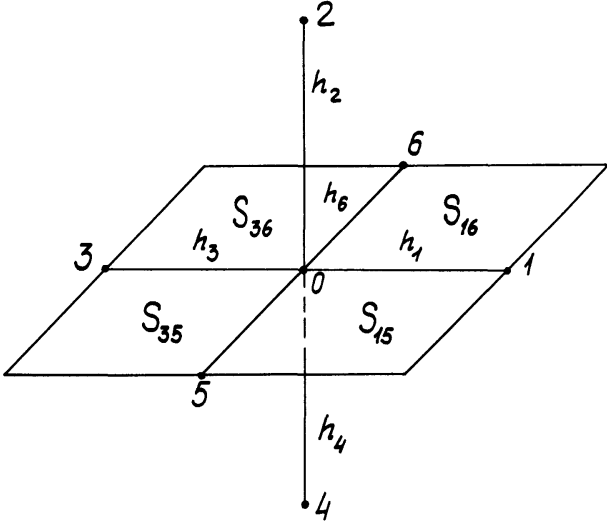

Fig. 6. - Seven-point pattern for determination of a scalar potential.

where $\rho_{i}$ is a magnetic charge density $\rho$ in the $i$-th node, and $\Delta V_{i}$ is the volume.

Taking into account that in our case magnetic charges are represented in a form of simple layers with surface density $\sigma$, we get

$$
\rho_{i} \Delta V_{i}=\int_{\Delta S_{i}} \sigma(r, \varphi, z) \mathrm{d} S .
$$

Magnetic charges $\sigma=A+B_{\varphi}$ are distributed over the surfaces $r=$ const. and four elementary surfaces $S_{15}, S_{16}, S_{35}, S_{36}$ around the central node of the patters. Therefore the right part of the equation (1) will be

$$
\begin{aligned}
\rho_{0}=\frac{1}{\Delta V_{0}}\left(\int_{S_{15}} \sigma \mathrm{d} S\right. & +\int_{S_{16}} \sigma \mathrm{d} S+ \\
& \left.+\int_{S_{35}} \sigma \mathrm{d} S+\int_{S_{36}} \sigma \mathrm{d} S\right) .
\end{aligned}
$$

Here

$$
\Delta V_{0}=\frac{h_{1}+h_{3}}{2} \cdot \frac{h_{2}+h_{4}}{2} r_{0} \frac{h_{5}+h_{6}}{2}
$$

(see Fig. 6).

The integrals in (17) can be calculated as follows :

$$
\begin{aligned}
\int_{S_{15}} \sigma \mathrm{d} S & =\int_{z_{0}}^{0+\frac{h_{1}}{2}} \int_{\varphi_{0}}^{\varphi_{0}+\frac{h_{5}}{2}} r_{0}\left(A_{i}+B_{i} \varphi\right) \mathrm{d} \varphi \mathrm{d} z \\
& =r_{0} \frac{A_{i} h_{1} h_{5}+B_{i} h_{1} h_{5}}{4}\left(\varphi_{0}+\frac{h_{5}}{4}\right) .
\end{aligned}
$$

All the other components of (17) can be defined in a similar way. The final expression for is :

$$
\rho_{0}=\frac{\sigma_{15} h_{1} h_{5}+\sigma_{15} h_{1} h_{5}+\sigma_{35} h_{3} h_{5}+\sigma_{35} h_{3} h_{6}}{\left(h_{1}+h_{3}\right)\left(h_{2}+h_{4}\right)\left(h_{5}+h_{6}\right)} \text {. }
$$




\section{Some results of investigations.}

2.1 EVALUATION OF THE DESIGN EFFECTIVENESS BY MEANS OF TWO-DIMENSIONAL MODEL. - In figure 7 and figure 8 the values of axial component of $B_{z}$ are given. The values of $B_{z}$ were received numerically using the results of a two-dimensional mathematical model. The $1200 \mathrm{MW}, 3000 \mathrm{rpm}$ turbo-type generators are considered. The comparison of two different designs of the stator core end packets shows that the maximum values of $B_{z}$ for both designs are approximately the same but for the design with smaller angle (from the axis) of the end packets tapering the axial magnetic flux is distributed over the larger surface of the packets, what leads to higher losses therein.

The values of losses in the inner edge (situated closer to the stator core bore) of the stator core press plate are given in table I. From the table it follows that the losses caused by tangential and radial

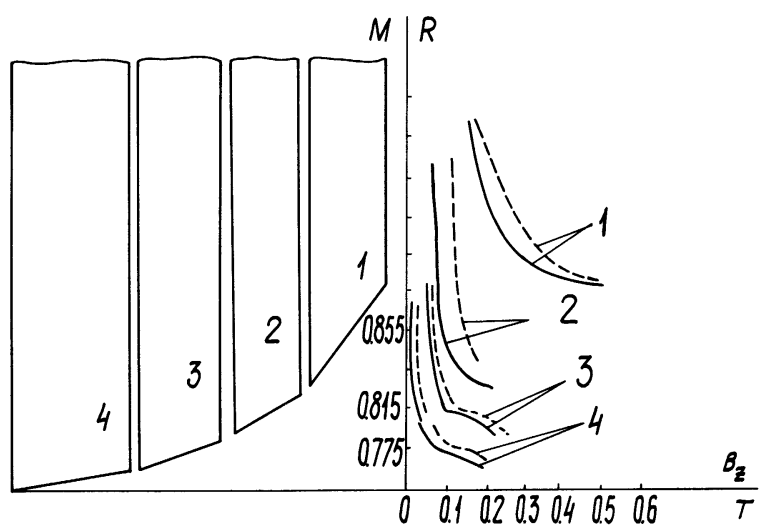

Fig. 7. - The distribution of axial $B$ component along the packet surfaces (small end packets tapering angle) : (solid line) rated load conditions; (dotted line) underexcited operation.

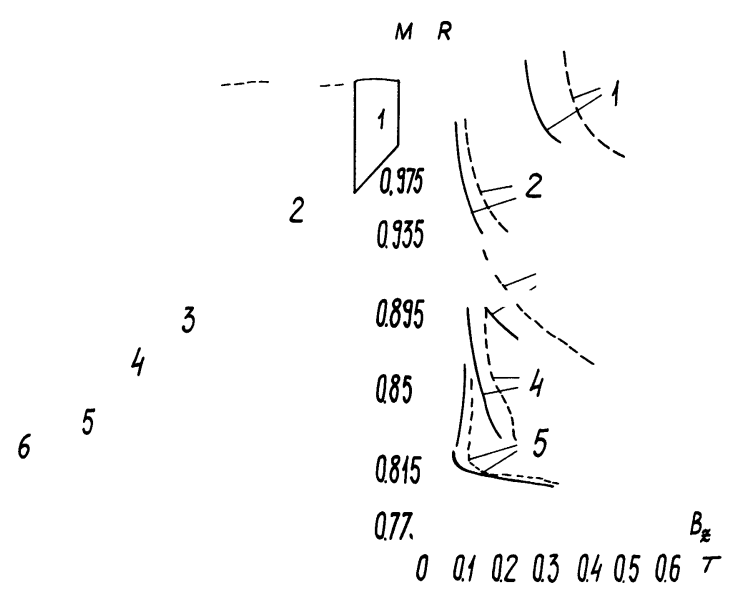

Fig. 8. - The distribution of axial $B$ component along the packet surfaces (large end packets tapering angle) : (solid line) rated load conditions; (dotted line) underexcited operation.
Table I. - Specific losses $P\left(\mathrm{~W} / \mathrm{cm}^{3}\right)$ in the inner edge surface of stator core press plate along the stator axis $\left(z_{1}<z_{2}<z_{3}\right)$.

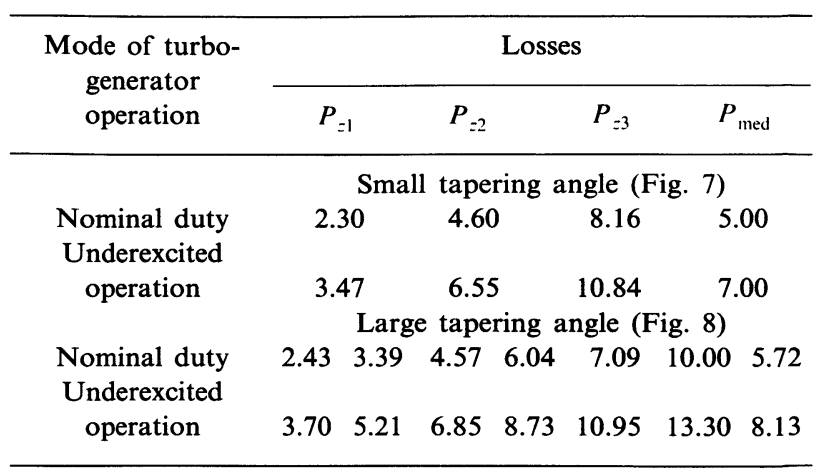

components of the eddy currents are approximately $15 \%$ greater for the design with larger angle of the end packets tapering. This is explained by a redistribution of the electromagnetic field in the end zone of the turbogenerator.

2.2 DETERMINATION OF THE MAXIMUM LOSSES AND TEMPERATURE RISES. - Three dimensional field investigation gives the possibility to determine the precise magnetic field distribution along the stator core in the tangential direction. It was found that the axial component of the magnetic induction $B_{z}$ within the winding phaze zone had more complex distribution than it was predicted from two-dimensional model, the main assumption of which is harmonic distribution of $B_{z}$ in tangential direction. According to the investigations, $B_{z}$ has the maximum on the end packet tooth surface situated on the joint of two stator winding phase zones of the lower winding layer. This maximum is observed when turbogenerator is in underexcited operation duty and is confirmed by increasing of about 20-30 $\mathrm{K}$ end packet temperature rise in comparison with other stator core teeth belonging to the same phase zone.

\section{Conclusion.}

Experience of the numerical modelling of the processes in the turbogenerator end zone showed that both two- and three-dimensional models were useful for the turbogenerator design features evaluation. When the axis-symmetrical elements are considered, e.g. press plates, screens, two-dimensional model provides quite adequate results. The data received from two-dimensional model consideration can be used as boundary conditions of the three-dimensional model by means of which more precise magnetic induction distribution along the turbogenerator stator core in tangential direction can be found. The last is of a great importance for the prediction of the largest possible temperature rises and thermomechanical stresses following from them. 


\section{Appendix 1.}

Let us determine the magnetic fields through $\bar{H}$ - magnetic field strength, which in its turn can be expressed through $\bar{H}_{0}$ - vector potential of the current and scalar magnetic potential

$$
\bar{H}=\bar{H}_{0}-\overline{\operatorname{grad}} \text {, }
$$

where $\bar{H}_{0}$ can be expressed in the only way through $\bar{y}$ ings

- current densities of the stator and rotor wind-

$$
\operatorname{rot} \bar{H}_{0}=\bar{g} \text {. }
$$

Using the equation for magnetic flux density $B$ divergence

$$
\operatorname{div} \bar{B}=\operatorname{div} \mu \bar{H}=0
$$

and substituting (A3) into (A1) we get

$$
\operatorname{div} \mu\left(\bar{H}_{0}-\overline{\operatorname{grad}} \cup\right)=0
$$

from where we shall have

$$
\text { or } \begin{aligned}
& \operatorname{div} \mu \overline{\operatorname{grad}} \vartheta=\operatorname{div} \mu \bar{H}_{0} \\
& \operatorname{div} \mu \overline{\operatorname{grad}} \vartheta=-\rho .
\end{aligned}
$$

In $(\mathrm{A} 6)$ it is denoted

$$
-\rho=\operatorname{div} \mu \bar{H}_{0} .
$$

\section{Appendix 2.}

Methods of determining vector $\boldsymbol{H}_{0}$ can be different. In this paper we used the representation of $\bar{H}_{0}$ as in $[4,5]$

$$
\bar{H}_{0}=\int_{\ell_{0}}^{\ell} g(\ell) \times \mathrm{d} \boldsymbol{l}
$$

where $\ell_{0}$ is the point, line or surface from the beginning of coordinates, $\mathrm{d} \bar{l}$ is the coordinate vector and $\bar{\gamma}(\ell)$ is the vector of current density.

When $\bar{H}_{0}$ is represented according to (A8) there is the possibility to choose $\bar{H}_{0}$ itself and $\ell_{0}$ and $\ell$ in a way which provides minimum volume of calculating scalar potential $\vartheta$.

\section{Appendix 3.}

Formulae for coefficients of magnetic charges $\sigma_{i}(\varphi)=A_{i}+B_{i}(\varphi)$ at the sections shown in figure 4 .

$$
\begin{aligned}
& A_{1}=-\frac{g_{\mathrm{II}}}{r_{1}} r_{2}^{2} \sin \varphi_{\mathrm{b}} ; \quad B_{1}=\frac{R_{1}^{2}}{r_{1}} g_{1} ; \\
& A_{2}=B_{1} \varphi_{1}-B_{2} \varphi_{2}-\frac{\gamma_{\mathrm{II}} r_{2}^{2}}{r_{1}} \sin \varphi_{\mathrm{b}} ; \quad B_{2}=\frac{R_{1}^{2}}{r_{1}} g_{2} ; \\
& A_{3}=\frac{g_{\mathrm{II}}}{r_{3}} r_{2}^{2} \sin \varphi_{\mathrm{b}} ; \quad B_{3}=-\frac{R_{2}^{2}}{r_{3}} g_{3} \text {; } \\
& A_{4}=B_{3} \varphi_{1}-B_{4} \varphi_{2}-\frac{\gamma_{\mathrm{II}}}{r_{3}} r_{2}^{2} \sin \varphi_{\mathrm{b}} ; \quad B_{4}=-\frac{R_{2}^{2}}{r_{3}} g_{4} \text {; } \\
& A_{5}=0 \text {; } \\
& B_{5}=\frac{R_{2}^{2}}{r_{2}} y_{3}-\frac{R_{1}^{2}}{r_{2}} \gamma_{1} \text {; } \\
& A_{6}=B_{5} \varphi_{1}-B_{6} \varphi_{2} \\
& B_{6}=\frac{R_{2}^{2}}{r_{2}} g_{4}-\frac{R_{1}^{2}}{r_{2}} y_{2} \text {; } \\
& A_{7}=B_{1} \varphi_{1}-\frac{\digamma_{\mathrm{II}}}{r_{1}} r_{2}^{2} \sin \varphi_{\mathrm{b}} ; \quad B_{7}=0 \\
& A_{8}=B_{1} \varphi_{1} \mu_{r}-\mu_{\mathrm{II}} \frac{\gamma_{\mathrm{II}}}{r_{1}} r_{2}^{2} \sin \varphi_{\mathrm{b}} ; \quad B_{8}=0 ; \\
& A_{9}=B_{3} \varphi_{1}+\frac{\digamma_{\mathrm{II}}}{r_{3}} r_{2}^{2} \sin \varphi_{\mathrm{b}} ; \quad B_{9}=0 \\
& A_{10}=B_{3} \varphi_{1} \mu_{r} ; \quad B_{10}=0 ; \\
& A_{11}=B_{5} \varphi_{1} ; \quad B_{11}=0 \text {; } \\
& A_{12}=B_{5} \varphi_{1} \mu_{r} ; \quad B_{12}=0 \text {; }
\end{aligned}
$$




$$
\begin{array}{lll}
A_{13}=-\mu_{\mathrm{II}} \frac{g_{\mathrm{II}}}{r_{1}} r_{2}^{2} \sin \varphi_{\mathrm{b}} ; & B_{13}=\frac{R_{1}^{2}}{r_{1}} g_{1} ; \\
A_{14}=B_{1} \varphi_{1}-B_{2} \varphi_{2}-\mu_{\mathrm{II}} \frac{g_{\mathrm{II}}}{r_{1}} r_{2}^{2} \sin \varphi_{\mathrm{b}} ; & B_{14}=\frac{R_{1}^{2}}{r_{1}} g_{2} ; \\
A_{15}=\mu_{\mathrm{II}} \frac{g_{\mathrm{II}}}{r_{3}} r_{2}^{2} \sin \varphi_{\mathrm{b}} ; & B_{15}=-\frac{R_{2}^{2}}{r_{3}} g_{3} ; \\
A_{16}=B_{3} \varphi_{1}-B_{4} \varphi_{2}+\mu_{\mathrm{II}} g_{\mathrm{II}} \frac{r_{2}^{2}}{r_{3}} \sin \varphi_{\mathrm{b}} ; & B_{16}=-\frac{R_{2}^{2}}{r_{3}} g_{4} ; \\
A_{17}=0 ; & B_{17}=\frac{R_{2}^{2}}{r_{2}} g_{3}-\frac{R_{1}^{2}}{r_{2}} g_{1} ; \\
A_{18}=B_{5} \varphi_{1}-B_{6} \varphi_{2} ; & B_{18}=\frac{R_{2}^{2}}{r_{2}} g_{1}-\frac{R_{1}^{2}}{r_{2}} g_{2} .
\end{array}
$$

\section{References}

[1] Jacobs D. A. H., Minors R. H., Myerscough C. J., Rollason M. L. J., Steel J. G., Calculation of losses in the end region of turbogenerators, Proc. IEE 124, N 4 (1977).

[2] Ito K., Tokumasu T., Nagano S., Tari M., DoI S., Simulation for design purposes of magnetic fields in turbine-driven generator end region, IEEE Trans. Power Apparatus Syst. PAS-99 (1980) 1586-1596.

[3] Biddlecombe C. S., Heighway E. A., Simkin J., Trowbridge C. W., Methods for eddy current computation in three dimensions, IEEE Trans. Magn. MAG-18 (1982) 492-497.

[4] Demirchian K. S., Chechurin W. L., Sarma M. S., Scalar potential concept for calculating the steady magnetic fields and eddy currents, IEEE Trans. Magn. 12 (1976) 1283-1290.

[5] VoldeK A. I., Danilevich Ya. B., KosachevsKy V. I., YAKovleV V. I., Electromagnetic processes in the end parts of electrical machines (book), in Russian, Energoatomizdat, Leningrad (1983). 